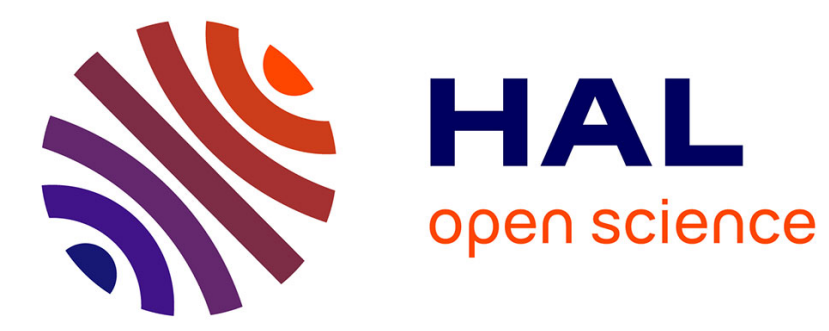

\title{
Review of Machine Learning Approaches In Fault Diagnosis applied to IoT System
}

Ndeye Gueye Lo, Jean-Marie Flaus, Olivier Adrot

\section{To cite this version:}

Ndeye Gueye Lo, Jean-Marie Flaus, Olivier Adrot. Review of Machine Learning Approaches In Fault Diagnosis applied to IoT System. ICCAD 2019 - 3rd International Conference on Control, Automation and Diagnosis, Jul 2019, Grenoble, France. 10.1109/ICCAD46983.2019.9037949 . hal-02344344

\section{HAL Id: hal-02344344 \\ https://hal.science/hal-02344344}

Submitted on 4 Nov 2019

HAL is a multi-disciplinary open access archive for the deposit and dissemination of scientific research documents, whether they are published or not. The documents may come from teaching and research institutions in France or abroad, or from public or private research centers.
L'archive ouverte pluridisciplinaire HAL, est destinée au dépôt et à la diffusion de documents scientifiques de niveau recherche, publiés ou non, émanant des établissements d'enseignement et de recherche français ou étrangers, des laboratoires publics ou privés. 


\title{
Review of Machine Learning Approaches In Fault Diagnosis applied to IoT System
}

\author{
Ndeye Gueye Lo*, Jean-Marie Flaus*, Olivier Adrot* \\ *Grenoble Alpes University, Grenoble Institute of Technology, CNRS, \\ G-SCOP Laboratory, Grenoble, France \\ Email: \{ndeye-gueye. lo, jean-marie.flaus, olivier.adrot\}@grenoble-inp.fr
}

\begin{abstract}
With increasing complex systems, low production costs, and changing technologies, for this reason, the automatic fault diagnosis using artificial intelligence (AI) techniques is more in more applied. In addition, with the emergence of the use of reconfigurable systems, AI can assist in self-maintenance of complex systems. The purpose of this article is to summarize the diagnosis research of systems using AI approaches and examine their application particularly in the field of diagnosis of complex systems. It covers articles published from 2002 to 2018 using Machine Learning tools for fault diagnosis in industrial systems.
\end{abstract}

Keywords - fault detection and diagnosis; artificial intelligence; machine learning; hybrid system; Internet of Things

\section{INTRODUCTION}

The current industrial trend regarding automatisms and regarding industrial plants leads us towards systems more and more complex mechatronics, working in an uncertain, evolutionary environment. It is so necessary to develop a diagnosis module to detect a fault (Fault Detection) that may affect the operation of these systems and to locate their causes (Fault Isolation). Therefore, a diagnosis module is needed to improve the performance and productivity of systems and limit the consequences of failures that can be catastrophic on human goods and life.

Diagnosis consists of detecting abnormal functioning from sensor data. These data may be noisy or corrupt due to unpredictable events. That abnormal operation may be a failure of process equipment (a sensor, actuator or a component), control system failure (due to operator error or cyber-attack of the system), or change of environment for example resources that are lacking (unavailable operators, exhausted stocks, etc.), or change due to non-conformity product etc. After detecting abnormal functioning, the cause can then be located and identified to make decisions (corrective actions or reconfiguration of the system). The different type of faults in the process is illustrated on the fig. 1.

In the literature, there are two main approaches for fault diagnosis, it is shown on the fig.2. : approaches that use an analytic or physical model of the system [1] and approaches that rely only on system observations [2]. The use of the techniques of diagnosis with models seems difficult and expensive, by offering less satisfactory performances. Besides, several industrial applications exist where a model is difficult even impossible to obtain due to increased complexity or several reconfigurations involved in the production process [3].

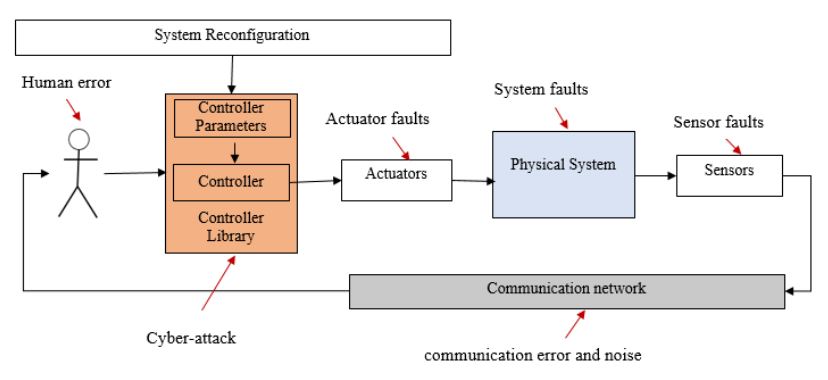

Fig. 1. Possible faults in the Cyber-Physical System

For this type of industrial application, the only operational monitoring methods are those without analytic or physical models. Among these process history-based techniques, we have signal processing tools and AI tools. However, the approach of signal processing tools is limited to detection and have huge problems with false alarms because they use statistical tests (mean, or variance) to define detection thresholds [4]. In fact, the artificial intelligence offers tools totally decoupled by the structure of the system, not requiring the preliminary modeling of the latter and allowing a real-time follow-up of its evolution. Besides, on-line reasoning makes that the approach of Artificial intelligence is stronger in changes in operating modes and have several reconfigurations.

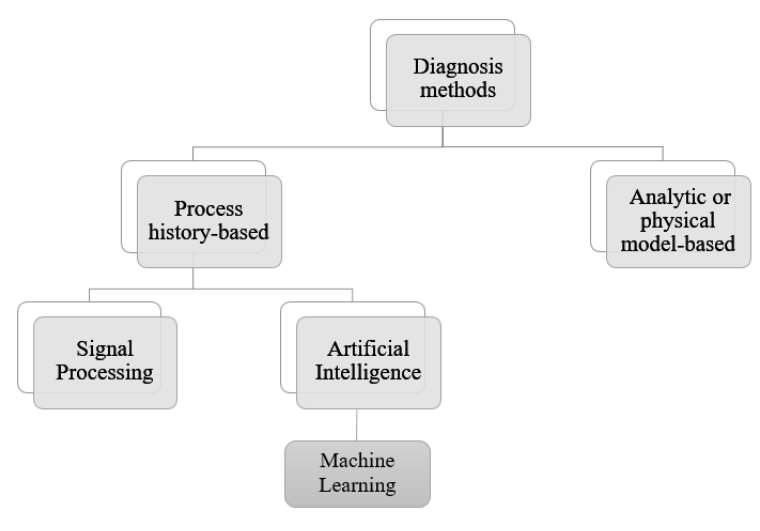

Fig. 2. The different methods in fault diagnosis 
Manufacturing is a mature industry with machines and assembly lines that run with a high degree of automation. Supervisory Control \& Data Acquisition Systems (SCADA) is prevalent industry standards and IoT is complementary to SCADA. SCADA's focus is on monitoring and control. IoT's focus is firmly on analyzing machine data to improve the productivity. SCADA systems consist of Programmable Logic Controllers (PLC) for interfacing with sensors in the machines. It is illustrated on the fig.3. IoT and SCADA are complementary technologies for Industry 4.0.

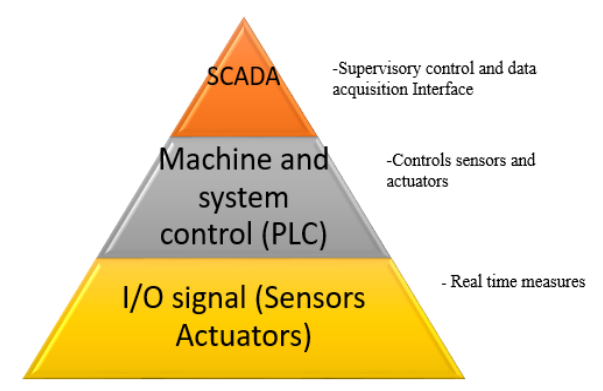

Fig. 3. The different layers in SCADA system

In the era of Industry 4.0, the Internet of Things (IoT) technologies is applied in many production systems. One of the important applications of IoT systems in the industry is monitoring, fault detection, and diagnosis of manufacturing systems [5]. Most of the IoT systems involve many sensors that collect a huge quantity of data and machine learning (ML) methods are applied to analyze them for various goals [6]-[7]. ML can be defined as a field of study in computer science that enables personal computers to automatically get more efficient at a given task through experience [8]. In fact, the interesting use of machine learning tools is that they can process many data and are tools of decision-making support for fault diagnosis [9].

The organization of this paper is as follows. After the introduction in Section I, some issues in fault diagnosis are presented in Section II. The machine learning approaches in fault diagnosis are reviewed in Section III. In Section IV we discuss the issues presented in the introduction. This paper is ended in Section $\mathrm{V}$ with a conclusion on the challenge of machine learning tools in the diagnosis and future research.

\section{SOME ISSUES IN FAULT DIAGNOSIS}

The main problem detection is the setting of a threshold. In fact, setting a low threshold for detection, generate many false alarms. These could disrupt the production system by causing production delays and affect planning and logistics. On the other hand, setting a very high detection threshold may result in the risk of not detecting a slow drift or serious situations. This may not be tolerated for operational safety and security issues. In this context, there are many uncertainties to consider: uncertainties related to lack of process knowledge, data uncertainties in sensors that may be noisy or missing data, uncertainties related to process variability.
In this context, where the system becomes very complex and it is hard to define an analytic model of the system, ML models are used to cope with this challenge. However, the main issues of ML tools are the data quality (missing or noisy data), the ability to consider several types of variables (discrete or continuous), the way to consider the time for the dynamic system and the ability to generalize in order the model has the capacity to take a good decision with a new observation.

\section{MACHINE LEARNING APPROACHES}

The application of IoT systems in industries creates a huge amount of data. In addition, these industrial systems have become more and more complex and it is difficult to obtain an analytical model of the system. In this context, the use of ML tools comes out obvious and logic to cope with the challenges of diagnosis in these systems. The goal of this paper is to show through several methods, the application of ML techniques on fault detection and diagnosis problems. Most of the publications reviewed and cited in this paper cover articles published from 2002 to 2018 using ML tools for fault diagnosis in industrial systems. ML methods are reviewed in the literature many times [10]-[11]. Therefore, we will review the most important and popular ML techniques in the diagnosis of a process in industries. Among the machine learning techniques, there are Support Vector Machine (SVM), Artificial Neural Network (ANN), Fuzzy Neural Network (FNN), Decision Trees (DT), Bayesian Network (BN).

\section{A. Support Vector Machine}

SVM [12] has an excellent performance in generalization with small training data, it is applied in an analog circuit fault diagnosis using wavelet transform as preprocessor with high accuracy in classification [13]. The idea of using SVM for separating two classes is to find support vectors to define the bounding planes, in which the margin between both planes is maximized. The number of support vectors increases with the complexity of the problem. However, SVM is less good when you have more than ten classes. In fact, in [14] proximal support vector machines (PSVM) reduce significant computation time and memory requirement in fault diagnosis on the Tennessee Eastman process (TEP) simulator when we have multiple fault classes. Recently, semi-supervised data-driven fault detection and diagnosis algorithm are ventilation air-conditioning (HVAC) faults acquiring only a few faulty training samples in [15]. The proposed semi-supervised SVM solves the problem of not enough faulty training samples with high performance proposed to detect and diagnose the components of heating.

SVM tends to generalize well even with a limited amount of training data. The main challenge is to select the kernel function parameters is very difficult. In addition, it is less efficient in a case of lot of data, because number of support vectors increases with the complexity of the problem.

\section{B. Artificial Neural Network}

ANN are powerful tools with the ability to perform classification and prediction operations. ANN can be considered as weighted directed graphs, the neurons being the nodes, and the connections between the nodes being weighted links. This learning adjusts weights as well as activation functions to adopt the desired behavior. Two types of learning are used: supervised learning and unsupervised learning [16]. 
-Supervised learning: the aim is to determine synaptic weights from the tagged examples. Thus, the network parameters are modified to minimize the error between the target output (provided by the expert) and the actual output of the network. Backpropagation is an example of a supervised learning algorithm [17].

-Unsupervised learning: the input data does not contain information about the desired output; the learning is carried out with rules that change the parameters of the network according to the input data. The associative memory learning algorithm for Hopfield Nets is an example [18].

In [18] quantum Hopfield Nets is used to diagnose the multiple faulty behaviors of analog circuits using probability mechanism. The fault patterns are then quantized and standardly orthogonalized to fed to the quantum Hopfield neural network. Using a quantum associative memory mechanism and quantum superposition principle, the systematic approach gives a good explanation of the probability of multiple faults. In [19], neural network-based fault detection and isolation (FDI) scheme is presented to detect and isolate faults in a highly nonlinear dynamics of an aircraft jet engine. The fault detection and isolation schemes consist of multiple ANN or parallel bank of filters, corresponding to various operating modes of the healthy and faulty engine conditions. Using the residuals that are generated by measuring the difference of each network output and the measured engine output various criteria are established for accomplishing the fault diagnosis task, that is addressing the problem of fault detection and isolation of the system components. A novel approach proposed in [20], is to create a neural model to emulate normal system behavior and additional models to emulate various fault conditions. The neural models are then placed in parallel with the system to be monitored, and fault detection is achieved by comparing the outputs of the neural models with the real system outputs. Fault classification is based on a simple threshold test of the residuals formed by subtracting each neural model output from the corresponding output of the real system. A Bayesian network is used to evaluate the residuals.

The power of ANN is their ability to approximate and recognize patterns. In diagnostic applications they have shown great promise in areas where noise and error are present. However, ANN is computationally intensive making convergence typically slow during training and prone to overfitting and requires a large diversified dataset for training to prevent this problem.

\section{Fuzzy Neural Network}

Diagnosis applications mainly include hybrid neuro-fuzzy models, which neural networks and fuzzy systems are combined homogeneously. The most common neuro-fuzzy systems are based on two types of models, the Tagaki Sugeno Kang (TSK) and Mamdani models [21], combined with neural learning algorithms. The Neuro-Fuzzy classification (NEFCLASS) is also recognized for these classification capabilities [22]. There are two main uses of neuro-fuzzy networks in monitoring. These uses are most often based on the study of residues that are generated by the difference of an estimated signal given by a neuro-fuzzy observer with the actual values of the signal. These residues are then classified and evaluated by the neurofuzzy system.
In [23], a neuro-fuzzy based learning and adaptation of TSK fuzzy models are used for residual generation, while for residual evaluation a neuro-fuzzy classifier for Mamdani models is used. The purpose is to detect and isolate faults to an industrial gas turbine, with emphasis on faults occurred in the actuator part of the gas turbine. An intelligent method is presented in [24] for fault diagnosis based on fault tree analysis and a fuzzy neural network in IoT equipment used for aquaculture. The fault tree presents a logic structure of fault symptoms and faults. Rules extracted from the fault trees avoid duplicate and redundancy. Moreover, the fuzzy neural network is applied to train the relationship mapping between fault symptoms and faults. The results show that this method can diagnose multiple faults with accuracy. Sequential Fuzzy Clustering Dynamic based Fuzzy Neural Networks is developed in [25] and successfully applied for monitoring of a high-speed milling process. It can sequentially learn the model, adapt itself to variations, and provide an estimation or prediction on the status of the process. It facilitates for nonintrusive fault diagnosis.

FNN has both advantages of ANN and fuzzy logic. In fact, it has the capacity to represent inherent uncertainties of the human knowledge with linguistic variables and it is robustness in relation of the possible disturbances in the system. Nevertheless, FNN need to have some expertise on the system to establish rules and the training time are very computationally intensive.

\section{Decision Trees}

The DT is designed to classify or predict a discrete category from the data. In the machine learning sense, the goal is to create a classification model (classification tree) that predicts the value of a target variable by learning simple decision rules inferred from the data features [26]. There exist several algorithms for training decision trees from data including ID3, C4.5, and CART. A novel mode estimation algorithm is developed in [27] that uses model-based prediction with Petri nets. And the diagnostic system traverses the decision-tree (ID3) and requests sensor tests as indicated by its nodes. The monitoring algorithm computes the deviations between the expected and the actual sensor values. This method is applied in a hybrid system, DC265 printer. The use of a decision tree offers efficiency; however, it limits the approach to the diagnosis of single faults. In [28], the authors propose to propagate wind turbine telemetry through a decision tree learning algorithm to detect faults, damage, and abnormal operations. They train a set Bagged decision tree classifier on a dataset from an offshore wind farm comprising 48 wind turbines and use it to automatically extract paths linking excessive vibrations faults to their possible root causes. CART algorithm is used for decision tree induction to a diagnostic strategy for air handling units in [29]. A steady-state detector and a regression model are incorporated into the strategy to increase the interpretability of the diagnostic strategy developed. It is shown that this strategy can achieve good diagnostic performance. In [30], the proposed method is based on Random Forest (RF), a novel ensemble classifier that builds many decision trees to improve the single tree classifier. This method is applied to motor faults diagnosis and it gives a good accuracy of faults classification. In [31], they propose to use the on-line random forests (ORF) algorithm to identify sensor fault. The sample set is derived from Tennessee Eastman process. Experiment results show that the accuracy of ORF is almost the same as RF and ORF has better adaptability than RF. 
DT are visually more intuitive, simpler and easier to assimilate and interpret by humans and engineers. Third, unlike other classification methods, with decision tree classifiers, one can perform data-driven root cause analysis of faults; one can trace a path from the end state to the initiating, a way that follows the sequence and chronology of how events are interlinked. They are very robust to noisy data and incomplete data. However, they need to use pruning parameter to reduce the overfitting.

\section{E. Bayesian Network}

$\mathrm{BN}$ is an important probabilistic graphical model, which can deal effectively with various uncertainty problems based on probabilistic information representation and inference. $\mathrm{BN}$ is a probabilistic graphical model representing a set of random variables and their conditional dependencies via a directed acyclic graph. Such a network consists of qualitative and quantitative parts. Qualitative part is directed acyclic graph, in which nodes represent system variables, whereas arcs symbolize dependencies or cause-and-effect relationships among variables. The quantitative part consists of the conditional probabilistic table, which represents the relationship between each node and its parents. Fault diagnosis procedures with BNs consist of $\mathrm{BN}$ structure modeling, BNs parameter modeling, BN inference, fault identification, and validation and verification. Several methods were reported in constructing BN structure models for fault diagnosis. Three main methods include cause-and-effect relationship, mapping algorithms, or structuring learning. Moreover, the parameters are the prior probability of root nodes and the conditional probability of leaf nodes. These probabilities can be achieved from expert knowledge and experience and statistical results of historical, simulated, and experimental data [32]. In [3], proposed algorithms are based on analyzing the observed behavior of the system and comparing it with a set of behavioral patterns generated based on various faulty conditions. They show how such a pattern matching problem can be formulated as an estimation of the posterior distribution of a Bayesian probabilistic model. When fault diagnosis is involved in temporal, system, or complex systems, inevitable difficulties with static BNs are observed. Therefore, some other types of BNs, such as Dynamic Bayesian Network (DBN) and Object-Oriented Bayesian Network (OOBN), are used to solve these problems. DBNs are extensional BNs with time-dependent variables and can be used to model the temporal evolution of dynamic systems. Thus, an approach relies on the use of Bayesian Network, which model the electrical power system to the arithmetic circuit, is proposed in [33]. This method can handle varying fault dynamic, fault progression and fault behavior cardinality. In [34], DBN are used to model the dynamic degradation process of electronic products, and Markov chains are used to model the transition relationships of four states, i.e., no fault, Transient Fault (TF), Intermittent Fault (IF), and permanent fault (PF). In addition, the fault diagnosis methodology can identify the faulty components and distinguish the fault types at a different time. OOBNs provide an approach to achieve hierarchical representation of the model, and each level corresponds to the level of abstraction, revealing encapsulated nodes for the current layer of an object. This approach reduces the complexity of building $\mathrm{BNs}$ and improves reusability of models.
A real-time fault diagnosis methodology of complex systems with repetitive structures is proposed using OOBN in [35]. Once faults occur, the proposed OOBN-based fault diagnosis system can report the faults and warnings. For a specified system with a certain situation, the operator can input some known experience information to the additional information layers of the additional information and common cause failure subnetworks.

$\mathrm{BN}$ is intuitively easy for the user to understand the interaction between the model variables. It is useful for modeling uncertainty and can be readily used to model hierarchical levels of multiple causes and effects with data from numerous sources, which is typically found in manufacturing systems. The main challenge of training a $\mathrm{BN}$ is in the construction of the tree structure and several methods including expert opinion have been proposed to this challenge.

\section{DISCUSSIONS}

In the diagnosis process, the system evolves in a nondeterministic environment that requires considering uncertainties that are noisy measurements, missing data, lack of knowledge of the system and its variability. Generally, the good performance of a model of machine learning results mainly from the quality of the data used. The main challenge of diagnostic systems in industrial applications is the estimation of faults with incomplete observations. In industrial practice, it is necessary to deal with incomplete datasets and unknown measurements, while continuously demanding useful and reliable information to support decision-making. The complexity of this issue depends on the mechanism of missing data and the informative level of the process database [36]. There are many approaches for solving these problems such as deletion of the incomplete or estimation of missing data. However, some ML tools can handle directly incomplete data. For example, BN could reason with incomplete observations. In fact, it uses probabilities to estimate our uncertainty degree and sometimes it uses expectation-maximization algorithms (EM) to learn parameters when it exists some missing data in the dataset [37].

An important part of ML is the ability of the model to generalize. Indeed, a model that is too complex relative to the problem we want to model will learn from the noise included in the dataset; therefore, the model may be overfitting, unable to generalize, and do many errors with a new observation. For this reason, in certain methods, pruning techniques are used, for example, decision trees and neural networks. The goal of pruning techniques is to stop the training to avoid overfitting. In addition, most of these methods are supervised techniques that use the expert's knowledge to make the diagnosis. The major drawback of supervised techniques is that the model only knows the type of faults it has learned. Nevertheless, with a new fault, some models can do ambiguity rejection or distance rejection to fill the lack of knowledge of the system and to adapt to the system evolution (useful for multiple reconfiguration systems). 
Furthermore, in the diagnosis process, the sensors generate numerical data, and not all ML tools use directly the numerical data. However, most machine learning techniques work only with continuous variables, for example, SVM, and ANN. On the other hand, other techniques use discrete variables; it is the case of DT and BN. In DT, C4.5 supports numerical target variables. For the other algorithms like ID3 and CART, they use discrete variables or convert the continuous variables into the interval. BN use usually discrete random variable and sometimes it is possible to incorporate random continuous variable if it has a Gaussian probability distribution [41]. FNN is the only technique that could handle various type of data (numeric and symbolic). In fact, it converts quantitative and qualitative values into a fuzzy set. The optimal diagnosis process is to build a model ability to process numerical or symbolic data where the operator may incorporate his knowledge by improving the diagnosis. Finally, we summarize the discussion given the table I below that allows comparing techniques according to the type of variables used.

TABLE I. COMPARISON OF TECHNIQUES ACCORDING TO SOME CRITERIA

\begin{tabular}{|l|c|c|c|c|c|}
\hline \multirow{2}{*}{} & \multicolumn{5}{|c|}{ Techniques } \\
\cline { 2 - 6 } & $S V M$ & $A N N$ & $F N N$ & $D T$ & $B N$ \\
\hline $\begin{array}{l}\text { Discrete } \\
\text { value }\end{array}$ & - & - & + & + & + \\
\hline $\begin{array}{l}\text { Continuous } \\
\text { value }\end{array}$ & + & + & + & - & - \\
\hline $\begin{array}{l}\text { Ability to } \\
\text { generalize }\end{array}$ & + & + & + & - & - \\
\hline $\begin{array}{l}\text { Robustness to } \\
\text { missing data }\end{array}$ & - & + & + & + & + \\
\hline
\end{tabular}

Another issue in the diagnosis process is the setting of the detection threshold. For example, with BN, some researchers rely on the posterior probability of the fault node. Usually, the probability of occurrence of an event is high if its posterior probability increases. However, this way can lead to errors results as some failures may have high probabilities inherently before inference. To avoid this issue, some use fuzzy logic to reduce false alarm rates, for example, the FNN [40] or establish a set of rules based on probability in case of BN [32].

Variability of the process is useful to consider in fault diagnosis. In fact, the complex or hybrid systems are characterized by multiple reconfigurations, several operating modes, and the number of sensors changes regularly particularly in IoT Systems: addition or deletion of one or more sensors. The diagnosis of Hybrid Dynamic Systems (HDS) requires the joint exploitation of continuous dynamics and discrete dynamics [38]. In this case, to diagnose in this context, we will need robust tools with the change of models and measurement system. BN is usually applied in this type of systems in the literature [39].

\section{CONCLUSION}

This review of machine learning application in fault diagnosis demonstrates that ML techniques could be a very useful tool in fault detection and diagnosis. This study shows that a single method does not allow filling all the characteristics that one seeks in the diagnosis of a system. Some methods can complement others to have a better diagnosis system. In era industry 4.0, with the internet of things, sensors generate enormous volume, variety, and velocity of the data. Moreover, it is necessary to design a cloud for data storage. However, there are some issues such as security and scalability. Thus, it is important to build a system of diagnosis with the ability to detect and identify the fault due to a cyber-attack and the fault due to failure on the system. In the future, we will develop ML tools able to make a robust diagnosis and to cope with this challenge.

\section{ACKNOWLEDGMENT}

The authors would like to acknowledge the comments and suggestions of the various reviewers that have been incorporated in this revised manuscript.

\section{REFERENCES}

S. N. K. Venkat Venkatasubramanian, Raghunathan Rengaswamy, Kewen Yin, "A review of process fault detection and diagnosis Part I: Quantitative model-based methods," Comput. Chem. Eng., vol. 27, pp. 293-311, 2003.

[2] V. Venkatasubramanian, R. Rengaswamy, S. N. Kavuri, and K. Yin, "A review of process fault detection and diagnosis part III: Process history based methods," Comput. Chem. Eng., vol. 27, no. 3, pp. 327346, 2003.

[3] M. Najafi, D. M. Auslander, P. L. Bartlett, P. Haves, and M. D. Sohn, "Application of machine learning in the fault diagnostics of air handling units," Appl. Energy, vol. 96, pp. 347-358, 2012.

N. G. Lo, A. Soualhi, M. Frini, and H. Razik, "Gear and bearings fault detection using motor current signature analysis," in Proceedings of the 13th IEEE Conference on Industrial Electronics and Applications, ICIEA 2018, 2018.

[5] S. Sciences, "A systematic literature review in fault analysis for IoT Guru Prasad Bhandari * Ratneshwer Gupta," vol. X, no. January, pp. $1-18,2018$

[6] O. Niggemann, G. Biswas, J. S. Kinnebrew, H. Khorasgani, S. Volgmann, and A. Bunte, "Data-driven monitoring of cyberphysical systems leveraging on big data and the internet-of-things for diagnosis and control," CEUR Workshop Proc., vol. 1507, pp. 185-192, 2015.

[7] I. L. Yen, S. Zhang, F. Bastani, and Y. Zhang, "A Framework for IoT-Based Monitoring and Diagnosis of Manufacturing Systems," Proc. - 11th IEEE Int. Symp. Serv. Syst. Eng. SOSE 2017, pp. 1-8, 2017.

[8] M. Sharp, R. Ak, and T. Hedberg, "A survey of the advancing use and development of machine learning in smart manufacturing," $J$. Manuf. Syst., vol. 48, pp. 170-179, 2018.

[9] Z. Ge, Z. Song, S. X. Ding, and B. Huang, "Data Mining and Analytics in the Process Industry: The Role of Machine Learning," IEEE Access, vol. 5, pp. 20590-20616, 2017.

[10] T. T. Ademujimi, M. P. Brundage, and V. V. Prabhu, "A Review of Current Machine Learning Techniques Used in Manufacturing Diagnosis," IFIP Adv. Inf. Commun. Technol., vol. 513, pp. 407-415, 2017. 
[11] R. Liu, B. Yang, E. Zio, and X. Chen, "Artificial intelligence for fault diagnosis of rotating machinery: A review," Mech. Syst. Signal Process., vol. 108, pp. 33-47, 2018.

[12] Z. Yin and J. Hou, "Recent advances on SVM based fault diagnosis and process monitoring in complicated industrial processes," Neurocomputing, vol. 174, pp. 643-650, 2016.

[13] B. Long, J. Huang, S. Tian, and S. Province, "Least Squares Support Vector Machine Based Analog- Circuit Fault Diagnosis Using Wavelet Transform as," vol. 0, no. 2, pp. 1026-1029, 2008.

[14] L. H. Chiang, M. E. Kotanchek, and A. K. Kordon, "Fault diagnosis based on Fisher discriminant analysis and support vector machines," Comput. Chem. Eng., vol. 28, no. 8, pp. 1389-1401, 2004.

[15] K. Yan, C. Zhong, Z. Ji, and J. Huang, "Semi-supervised learning for early detection and diagnosis of various air handling unit faults," Energy Build., vol. 181, pp. 75-83, 2018.

[16] W. G. Fenton, T. M. Mcginnity, and L. P. Maguire, "Fault Diagnosis of Electronic Systems Using Intelligent Techniques: A Review," IEEE Trans. Syst. Man, Cybern. Part C (Applications Rev., vol. 31, no. 3, pp. 269-281, 2001.

[17] T. Sorsa, H. N. Koivo, and H. Koivisto, "Neural Networks in Process Fault Diagnosis," IEEE Trans. Syst. Man Cybern., vol. 21, no. 4, pp. 815-825, 1991.

[18] P. Li, Y. Chai, M. Cen, Y. Qiu, and K. Zhang, "Multiple fault diagnosis of analog circuit using quantum hopfield neural network," 2013 25th Chinese Control Decis. Conf. CCDC 2013, pp. 4238-4243, 2013

[19] S. Sina Tayarani-Bathaie, Z. N. Sadough Vanini, and K. Khorasani, "Dynamic neural network-based fault diagnosis of gas turbine engines," Neurocomputing, vol. 125, pp. 153-165, 2014.

[20] and K. S. L. Hyun Cheol Cho, Jeremy Knowles, M. Sami Fadali, "Fault Detection and Isolation of Induction Motors Using Recurrent Neural Networks and Dynamic Bayesian Modeling," in IEEE Transactions on Control Systems Technology, 2010, vol. 18, no. 2.

[21] K. V. Shihabudheen and G. N. Pillai, "Recent advances in neurofuzzy system: A survey," Knowledge-Based Syst., vol. 152, pp. 136$162,2018$.

[22] D. Nauck, U. Nauck, and R. Kruse, "Generating classification rules with the neuro-fuzzy system NEFCLASS," pp. 466-470, 2002.

[23] V. Palade, R. J. Patton, F. J. Uppal, J. Quevedo, and S. Daley, "Fault diagnosis of an industrial gas turbine using neuro-fuzzy methods," IFAC Proc. Vol., vol. 15, no. 1, pp. 471-476, 2002.

[24] Y. Chen, Z. Zhen, H. Yu, and J. Xu, “Application of fault tree analysis and fuzzy neural networks to fault diagnosis in the internet of things (IoT) for aquaculture," Sensors (Switzerland), vol. 17, no. 1,2017.

[25] A. Torabi Jahromi, M. J. Er, X. Li, and B. S. Lim, "Sequential fuzzy clustering based dynamic fuzzy neural network for fault diagnosis and prognosis," Neurocomputing, vol. 196, pp. 31-41, 2016.

[26] S. Piechowiak, "Intelligence artificielle et diagnostic," Électronique - Autom. | Autom. ingénierie système, vol. 33, no. 0, pp. 0-20, 2003.

[27] F. Zhao, X. Koutsoukos, H. Haussecker, J. Reich, and P. Cheung, "Monitoring and fault diagnosis of hybrid systems," IEEE Trans. Syst. Man, Cybern. Part B Cybern., vol. 35, no. 6, pp. 1225-1240, 2005.

[28] H. Mylonas et al., "Fault diagnosis of wind turbine structures using decision tree learning algorithms with big data," Saf. Reliab. - Safe Soc. a Chang. World, no. June, pp. 3053-3061, 2018.

[29] R. Yan, Z. Ma, Y. Zhao, and G. Kokogiannakis, "A decision tree based data-driven diagnostic strategy for air handling units," Energy Build., vol. 133, pp. 37-45, 2016.

[30] B. S. Yang, X. Di, and T. Han, "Random forests classifier for machine fault diagnosis," J. Mech. Sci. Technol., vol. 22, no. 9, pp. 17161725, 2008.

[31] L. Yang, J. Zhang, F. Deng, and J. Chen, "Sensor fault diagnosis based on on-line random forests," Chinese Control Conf. CCC, vol. 2016-August, pp. 4089-4093, 2016.
Diagnosis," IEEE Trans. Ind. Informatics, vol. 13, no. 5, pp. 2227 2240, 2017

[33] B. Ricks and O. J. Mengshoel, "International Journal of Approximate Reasoning Diagnosis for uncertain , dynamic and hybrid domains using Bayesian networks and arithmetic circuits," Int. J. Approx. Reason., vol. 55, no. 5, pp. 1207-1234, 2014.

[34] B. Cai, Y. Liu, S. Member, and M. Xie, "A Dynamic-BayesianNetwork-Based Fault Diagnosis Methodology Considering," vol. 14, no. 1, pp. 276-285, 2017.

[35] B. Cai, H. Liu, and M. Xie, "A real-time fault diagnosis methodology of complex systems using object-oriented Bayesian networks," Mech. Syst. Signal Process., vol. 80, pp. 31-44, 2016.

[36] M. Askarian, G. Escudero, M. Graells, R. Zarghami, F. JalaliFarahani, and N. Mostoufi, "Fault diagnosis of chemical processes with incomplete observations: A comparative study," Comput. Chem. Eng., vol. 84, pp. 104-116, 2016.

[37] Y. Wang, H. Yang, X. Yuan, and Y. Cao, "An improved Bayesian network method for fault diagnosis," IFAC-PapersOnLine, vol. 51 , no. 21, pp. 341-346, 2018.

[38] M. S. Mouchaweh and D. Hybrides, "Diagnostic des Systèmes dynamiques hybrides ( SDH )," Tech. l'Ingénieur, vol. 33, no. 0, 2015.

[39] G. Zhou, G. Biswas, W. Feng, H. Zhao, and X. Guan, “A Bayesian framework for fault diagnosis of hybrid linear systems," CEUR Workshop Proc., vol. 1507, pp. 27-34, 2015.

[40] R. Alshammari, S. Sonamthiang, M. Teimouri, and D. Riordan, "Using neuro-fuzzy approach to reduce false positive alerts," Proc. CNSR 2007 Fifth Annu. Conf. Commun. Networks Serv. Res., pp. 345-349, 2007.

[41] Y.-C. Chen, T. A. Wheeler, and M. J. Kochenderfer, "Learning Discrete Bayesian Networks from Continuous Data," vol. 59, pp. 103-132, 2015 